\title{
Francisella tularensis Modulates a Distinct Subset of Regulatory Factors and Sustains Mitochondrial Integrity to Impair Human Neutrophil Apoptosis
}

\author{
Jenna M. McCracken ${ }^{a, b}$ Lauren C. Kinkead ${ }^{a, b}$ Ramona L. McCaffrey ${ }^{a, c}$ \\ Lee-Ann H. Allen ${ }^{\mathrm{a}-\mathrm{c}}$ \\ ${ }^{\mathrm{a}}$ Inflammation Program, and Departments of ${ }^{\mathrm{b}}$ Microbiology and ${ }^{\mathrm{C}}$ Internal Medicine, University of lowa and \\ VA Medical Center, lowa City, lowa, USA
}

\section{Key Words}

Apoptosis · Mitochondria $\cdot$ Neutrophils $\cdot$ Proliferating cell nuclear antigen $\cdot \mathrm{X}$-linked inhibitor of apoptosis protein .

R-roscovitine

\begin{abstract}
Tularemia is a disease characterized by profound neutrophil accumulation and tissue destruction. The causative organism, Francisella tularensis, is a facultative intracellular bacterium that replicates in neutrophil cytosol, inhibits caspase activation and profoundly prolongs cell lifespan. Here, we identify unique features of this infection and provide fundamental insight into the mechanisms of apoptosis inhibition. Mitochondria are critical regulators of neutrophil apoptosis. We demonstrate that $F$. tularensis significantly inhibits Bax translocation and Bid processing during 24-48 h of infection, and in this manner sustains mitochondrial integrity. Downstream of mitochondria, X-linked inhibitor of apoptosis protein (XIAP) and proliferating cell nuclear antigen (PCNA) inhibit caspase- 9 and caspase- 3 by direct binding. Notably, we find that PCNA disappeared rapidly and selectively from infected cells, thereby demonstrating that it is not essential for neutrophil survival, whereas upregulation of calpastatin corre-
\end{abstract}

lated with diminished calpain activity and reduced XIAP degradation. In addition, R-roscovitine is a cyclin-dependent kinase inhibitor developed for the treatment of cancer; it also induces neutrophil apoptosis and can promote the resolution of several infectious and inflammatory disorders. We confirm the ability of R-roscovitine to induce neutrophil apoptosis, but also demonstrate that its efficacy is significantly impaired by $F$. tularensis. Collectively, our findings advance the understanding of neutrophil apoptosis and its capacity to be manipulated by pathogenic bacteria.

(c) 2016 S. Karger AG, Basel

\section{Introduction}

Polymorphonuclear leukocytes (PMNs, neutrophils) are essential effector cells of the innate immune system and serve as a first line of defense against infection. These cells are rapidly recruited to sites of infection and kill microbes, using a combination of toxic oxidants, proteases and antimicrobial peptides [1]. Unlike other leukocytes, PMNs are also very short-lived and are programmed to undergo constitutive (i.e. spontaneous and physiological) apoptosis within $24 \mathrm{~h}$ of release into the bloodstream,

\section{KARGER}

E-Mail karger@karger.com

www.karger.com/jin
(C) 2016 S. Karger AG, Basel

1662-811X/16/0083-0299\$39.50/0
Dr. Lee-Ann H. Allen

Inflammation Program, University of Iowa

2501 Crosspark Rd, MTF D-154

Coralville, IA 52241 (USA)

E-Mail lee-ann-allen@ uiowa.edu 
necessitating the production of approximately $10^{11}$ new neutrophils each day in humans [2]. At the same time, neutrophil lifespan is influenced by internal and external environmental cues including cytokines, reactive oxygen species and microbes [2-5]. Notably, phagocytosis accelerates PMN apoptosis, and this phagocytosis-induced cell death (PICD) response is critical for elimination of infection and resolution of inflammation $[2,4,5]$. During this process, neutrophil proinflammatory capacity is downregulated, release of histotoxic cell content is prevented and delivery of dying neutrophils to macrophages for disposal is enhanced $[2,5]$. Accordingly, this process is tightly regulated, and it is established that defects in PMN turnover define inflammatory responses that are aberrant and ineffective [6].

Francisella tularensis is a Gram-negative, facultative, intracellular, bacterial pathogen that infects several cell types, including neutrophils, and is the causative agent of tularemia. The pneumonic form of this disease, which can ensue following inhalation of as few as 10 bacteria, is the most severe form and has a $30-60 \%$ case fatality rate if left untreated [7]. Key features of pneumonic tularemia include profound accumulation of neutrophils and necrotic debris, such that air is excluded from the alveolar sacs and granulomatous foci form regionally $[8,9]$. In keeping with this, neutrophils account for $50 \%$ of infected cells in the lungs by the third day of infection, yet appear to contribute to disease pathology rather than effective host defense as blockade of neutrophil influx into the lungs significantly improves survival, whereas neutrophilia exacerbates tissue destruction and disease [10-14]. The extreme virulence of $F$. tularensis suggests that this pathogen has evolved robust strategies for evading host defense. Relevant here is the multifaceted strategy that $F$. tularensis uses to evade elimination by human neutrophils, which includes rapid disruption of NADPH oxidase assembly and activation, and is followed by phagosome escape and bacterial replication in PMN cytosol [15-17]. In addition, we recently demonstrated that $F$. tularensis not only fails to induce PICD, it also inhibits constitutive PMN apoptosis and, as a result, neutrophil lifespan is profoundly prolonged [17]. Accordingly, our data provide insight into the mechanisms that contribute to the profound neutrophil accumulation and tissue destruction that occurs during tularemia.

In the past decade, our understanding of the mechanisms that regulate constitutive and induced neutrophil apoptosis has greatly increased, and it is now clear that PMN viability and death are controlled via effects on gene expression as well as multiple intracellular signaling path- ways $[2,4,18,19]$. Our published data demonstrate that F. tularensis infection impairs processing and activation of caspase- 3 , caspase- 8 and caspase- 9 , and also significantly alters the neutrophil transcriptome, including the expression of 365 unique genes linked to apoptosis and cell survival $[17,20]$. Here, we expand our previous work to demonstrate that $F$. tularensis acts at multiple levels to sustain mitochondrial integrity and modulate cytosolic regulatory factors that act distal to mitochondria to inhibit caspase activity. At the same time, we also demonstrate that $F$. tularensis significantly undermines the efficacy of R-roscovitine, an apoptosis-inducing agent and candidate therapeutic developed for the treatment of cancer as well as inflammatory and infectious disorders that are characterized by defects in PMN turnover [21, 22]. Thus, this study advances our understanding of both PMN apoptosis and tularemia pathogenesis.

\section{Materials and Methods}

\section{Materials}

Cysteine heart agar, Bacto Brain Heart Infusion, rabbit antiF. tularensis antiserum, mouse anti-human Fas IgM (clone $\mathrm{CH}$ 11-activating) and antibodies specific for X-linked inhibitor of apoptosis protein (XIAP), Mcl-1 and Bax were obtained from Becton Dickinson (Sparks, Md., USA). Additional Mcl-1antibodies were from Proteintech (Chicago, Ill., USA). Defibrinated sheep blood was from Remel (Lenexa, Kans., USA). Clinical grade dextran (m.w. 500,000) was from Pharmacosmos A/S (Holbaek, Denmark) and Ficoll-Paque Plus was from GE Healthcare Biosciences (Uppsala, Sweden). Endotoxin-free Dulbecco's PBS, Hanks' balanced salt solution (HBSS) and HEPES buffer were from Mediatech Inc. (Manassas, Va., USA). Endotoxin-free HEPES-buffered RPMI-1640 was from Lonza (Walkersville, Md., USA). HEMA-3 staining kits were from Fisher Scientific (Kalamazoo, Mich., USA). Caspase-Glo assay kits were from Promega (Madison, Wis., USA). Annexin V-FITC and MitoProbe ${ }^{\mathrm{TM}} \mathrm{JC}-1$ assay kits were from Invitrogen (Eugene, Oreg., USA). Propidium iodide (PI) was from BioVision (Milpitas, Calif., USA). Pierce SuperSignal West Femto enhanced chemiluminescent substrate reagents, Pierce BCA protein assay kits and antibodies specific for manganese superoxide dismutase (MnSOD) were from Thermo Scientific (Rockford, Ill., USA). Anti-calpain-I ( $\mu$-calpain) antibodies were from Millipore (Temecula, Calif., USA). Antibodies specific for Smac/Diablo, calpastatin, Bid, Htra2/Omi and proliferating cell nuclear antigen (PCNA) were from Cell Signaling Technologies (Danvers, Mass., USA). Anti- $\beta$-actin antibodies were from BioLegend (San Diego, Calif., USA). Anti-TOM20 antibodies were from Santa Cruz Biotechnology (Santa Cruz, Calif., USA). Secondary $\mathrm{F}\left(\mathrm{ab}^{\prime}\right)_{2}$ antibodies conjugated to Alexa 488 and Dylight 549 were from Jackson ImmunoResearch Laboratories (West Grove, Pa., USA) and secondary antibodies conjugated to horseradish peroxidase were from BioRad (Hercules, Calif., USA). Staurosporine (STS), protease inhibitors and all other reagents were from Sigma-Aldrich (St. Louis, Mo., USA), unless otherwise specified. 


\section{Cultivation of F. tularensis}

The $F$. tularensis subspecies, $F$. holarctica live vaccine strain (LVS) [15], was grown on cysteine heart agar supplemented with $9 \%$ defibrinated sheep blood (CHAB) for $48 \mathrm{~h}$ at $37^{\circ} \mathrm{C}$ in $5 \% \mathrm{CO}_{2}$ as previously described $[15-17,20]$. Thereafter, bacteria were harvested from agar plates and subcultured in Brain Heart Infusion broth (pH 6.8) to mid-logarithmic phase as we have described [23]. Bacteria were collected by centrifugation, washed twice with HBSS containing divalent cations, and quantified by measuring the absorbance at $600 \mathrm{~nm}$. To test the possible effects of $20 \mu \mathrm{M}$ R-roscovitine on LVS growth, the drug was added to broth media, and bacterial growth was monitored by measurement of optical density and by plating serial dilutions on $\mathrm{CHAB}$ for the enumeration of colony-forming units.

\section{Neutrophil Isolation}

Heparinized venous blood was obtained from healthy adult volunteers in accordance with a protocol approved by the Institutional Review Board for Human Subjects at the University of Iowa, and all donors provided informed consent. Neutrophils were isolated from anticoagulated peripheral blood using dextran sedimentation followed by density gradient separation on FicollPaque Plus as described [24]. After hypotonic lysis of erythrocytes, PMNs were suspended in HBSS without divalent cations, counted and adjusted to $2 \times 10^{7} / \mathrm{ml}$. The purity of our preparations was assessed by HEMA-3 staining [17] and was routinely $>96 \%$. Replicate experiments utilized neutrophils from independent donors.

\section{Infection and Treatment Conditions}

Neutrophils in HEPES-buffered RPMI-1640 media were added to 5 - $\mathrm{ml}$ polypropylene tubes at $5 \times 10^{6} / \mathrm{ml}$ in the presence or absence of LVS at a ratio of $200: 1$, and then incubated at $37^{\circ} \mathrm{C}$ with $5 \% \mathrm{CO}_{2}$ for up to $48 \mathrm{~h}$ as we have previously described $[17,20]$. As indicated, some experiments utilized neutrophils that were treated with $50 \mu \mathrm{M}$ carbonyl cyanide m-chlorophenyl hyrazone (CCCP), $1 \mu \mathrm{M}$ STS, $500 \mathrm{ng} / \mathrm{ml}$ mouse anti-Fas IgM (clone CH-11) [17] or 20 $\mu \mathrm{M}$ R-roscovitine prior to analysis.

\section{Quantitation of Apoptosis by Flow Cytometry}

Neutrophil apoptosis was quantified using Annexin V-FITC to detect externalized phosphatidylserine and PI to detect plasma membrane disruption as we have described [17] with minor modifications. In brief, neutrophils were costained with Annexin $\mathrm{V}$-FITC and PI in AV-binding buffer (10 mM HEPES/NaOH, pH 7.4, $140 \mathrm{mM} \mathrm{NaCl}, 2.5 \mathrm{mM} \mathrm{CaCl}_{2}$ ) for $5 \mathrm{~min}$ prior to analysis using an Accuri C6 flow cytometer (BD Accuri Cytometers, Inc., Ann Arbor, Mich., USA). Five thousand events were collected for each sample and the data were analyzed using Accuri C6 software.

\section{Analysis of Nuclear Morphology}

Aliquots of $3.75 \times 10^{5}$ neutrophils were cytocentrifuged onto coverslips, fixed with $10 \%$ formalin for $15 \mathrm{~min}$ at room temperature and stained using HEMA-3 reagents according the manufacturer's instructions [17]. Stained neutrophils were examined by light microscopy and were scored as healthy if they contained a normal, 3- to 4-lobed nucleus, and as apoptotic when they exhibited condensed, spherical nuclei [17]. At least 200 cells per coverslip and condition were analyzed in each experiment.
Caspase-3 and Calpain Activity Assays

Caspase- 3 activity was measured using Promega Caspase-Glo kits according to the manufacturer's directions. Each assay contained $5 \times 10^{4} \mathrm{PMNs} /$ well in white microtiter plates. Luminescence generated by substrate peptide cleavage was detected using a NOVOstar luminometer (BMG LabTech, Cary, N.C., USA) [17]. To measure calpain activity in intact cells, $5 \times 10^{5} \mathrm{PMNs} / \mathrm{sample}$ were incubated with the fluorogenic peptide substrate Boc-LeuMet-CMAC (ThermoFisher Scientific) [25] at a 20- $\mu \mathrm{M}$ final concentration for $5-10 \mathrm{~min}$ at $37^{\circ} \mathrm{C}$, and fluorescence was detected using a POLARstar Omega microplate fluorometer (BMG Labtech).

\section{Mitochondrial Membrane Potential}

Neutrophils $\left(1 \times 10^{6}\right.$ per sample $)$ were pelleted and resuspended in warm HBSS without divalent cations and incubated for $5 \mathrm{~min}$ at $37^{\circ} \mathrm{C}$ prior to the addition of JC- 1 to a $2-\mu \mathrm{M}$ final concentration. After 30 min at $37^{\circ} \mathrm{C}$, cells were washed with warm HBSS without divalent cations and analyzed immediately by flow cytometry. The assay was validated using PMNs treated with $50 \mu \mathrm{M}$ CCCP. Ten thousand events were collected for each sample and the data were analyzed using Accuri C6 software.

\section{Immunoblotting}

Neutrophils were pelleted by centrifugation, resuspended in ice-cold PBS containing protease inhibitors (aprotinin, leupeptin, AEBSF, phenylmethylsulfonyl fluoride, levamisole, bestatin, E-64 and pepstatin A) and incubated on ice for $10 \mathrm{~min}$. Cells were disrupted by the addition of NP-40 lysis buffer to a $1 \%$ final detergent concentration, lysates were clarified by centrifugation, and protein concentrations were determined using the Pierce BCA assay. After boiling for $10 \mathrm{~min}$ in sample buffer containing reducing agent, equal amounts of each sample $(5-20 \mu \mathrm{g})$ were separated on $4-12 \%$ Bis-Tris NuPAGE gradient gels using MES running buffer, and then transferred to polyvinylidene fluoride membranes by electroblotting. Membranes were blocked with 5\% milk or 5\% BSA in PBS for $1 \mathrm{~h}$ and then probed to detect cytochrome $c$, Htra2, Smac, Bax, Bid, BCL2A1, Mcl-1, calpastatin, calpain, XIAP, PCNA, MnSOD, TOM20 or $\beta$-actin as indicated. After incubation with horseradish peroxidase-conjugated secondary antibodies and Pierce Supersignal West Femto enhanced chemiluminescence reagents, blots were imaged using either Kodak X-ray film or a LICOR Odyssey imaging system (LI-COR Biosystems, Lincoln, Nebr., USA). Immunoblots were quantified by densitometry [17]. These data are shown in online supplementary figure 1 (for all online suppl.material, see www.karger.com/doi/10.1159/000443882).

\section{Subcellular Fractionation}

Neutrophils $\left(1 \times 10^{6}\right)$ were incubated with $1 \mathrm{mM}$ diisopropyl fluorophosphate on ice for $20 \mathrm{~min}$ and then fractionated to separate cytosol and total membranes as described [26] with minor modifications. In brief, cells were pelleted and resuspended in ice-cold plasma membrane permeabilization buffer $(200 \mu \mathrm{g} / \mathrm{ml}$ digitonin, $80 \mathrm{mM} \mathrm{KCl}$ in PBS) supplemented with the protease inhibitor mixture described above, and then incubated on ice for $5 \mathrm{~min}$. This allows efficient and selective plasma membrane permeabilization mediated by interactions of digitonin with cholesterol. Next, cytosol was separated from the permeabilized cells by centrifugation at $800 \mathrm{~g}$ for $5 \mathrm{~min}$. The cytosol-depleted cell pellets (which contain mitochondria) were solubilized in ice-cold total cell lysis buffer [50 mM Tris- $\mathrm{HCl}$ (pH 7.4), $150 \mathrm{~mm} \mathrm{NaCl}, 2$ mM EGTA, 2 mM EDTA, 
Fig. 1. Apoptosis of infected neutrophils is impaired. The ability of mid-log phase $F$. tularensis to prolong PMN lifespan was quantified by Annexin V-FITC/PI staining and flow cytometry. a Representative flow cytometry plots of uninfected PMNs (top row) and LVS-infected PMNs (bottom row) obtained at 3, 6, 12 and $24 \mathrm{~h}$, as indicated. b Pooled flow cytometry data indicate the percentage of early apoptotic (Annexin $\mathrm{V}+/ \mathrm{PI}-)$ cells and late apoptotic/necrotic (Annexin $\mathrm{V}+/ \mathrm{PI}+$ ) cells at each time point and are the mean \pm SD of 6 independent experiments. ${ }^{* * *} \mathrm{p}<0.001{ }^{*} \mathrm{p}<0.05$. Where not visible, error bars are smaller than symbols. c Caspase- 3 activity in control, LVS-infected and STS-treated PMNs at each time point measured using Caspase-Glo reagents. Data are from 1 experiment representative of 5 independent determinations. $\mathrm{RLU}=$ Relative light units.
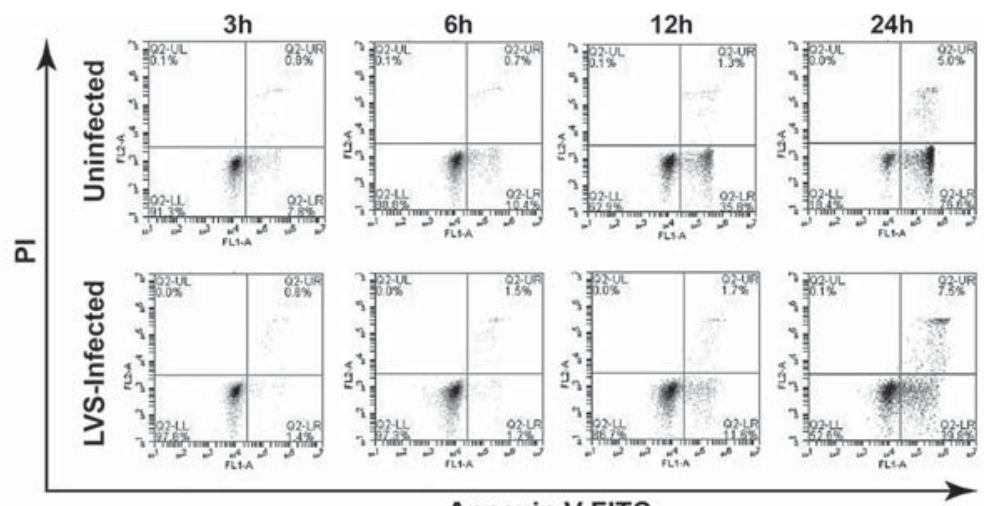

a

Annexin V-FITC
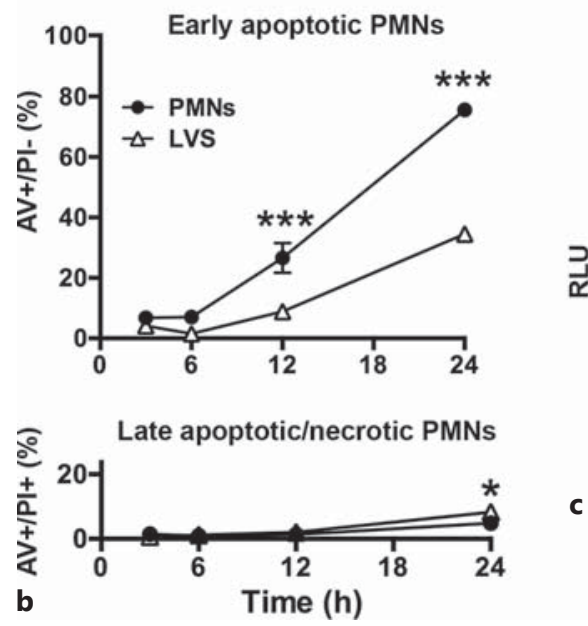

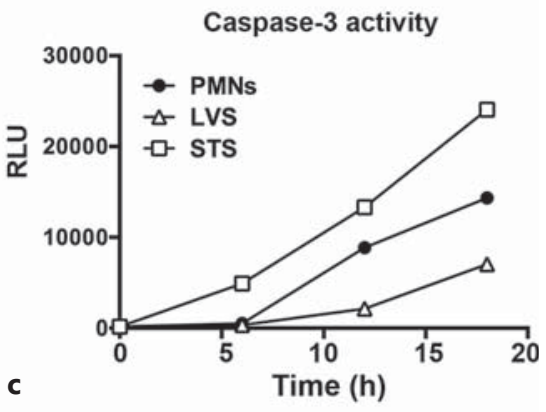

$0.2 \%$ Triton $\mathrm{X}-100,0.3 \% \mathrm{NP}-40$ in PBS] containing protease inhibitors, by gentle oscillation at $4^{\circ} \mathrm{C}$ for $10 \mathrm{~min}$, and then clarified by centrifugation at $10,000 \mathrm{~g}$ for $10 \mathrm{~min}$ to remove any insoluble material. The purity of the cytosolic and membrane fractions was assessed by immunoblotting using $\beta$-actin as a cytosolic marker and MnSOD or TOM20 as mitochondrial markers (data not shown).

Confocal and Transmission Electron Microscopy

At each time point, an aliquot containing $3.75 \times 10^{5}$ neutrophils was cytocentrifuged onto acid-washed glass coverslips. Cells were fixed with $10 \%$ formalin, permeablized with ice-cold methanolacetone (1:1) and blocked in buffer containing PBS, $0.5 \mathrm{mg} / \mathrm{ml}$ $\mathrm{NaN}_{3}$ and $5 \mathrm{mg} / \mathrm{ml} \mathrm{BSA}$, using our established methods [15, 27]. PMNs were stained to detect Bax alone or together with MnSOD or LVS using mouse monoclonal anti-Bax IgG, rabbit polycolonal antibodies specific for MnSOD and/or rabbit anti-LVS antiserum. Secondary $\mathrm{F}\left(\mathrm{ab}^{\prime}\right)_{2}$ antibodies were conjugated to Alexa Fluor-488 or DyLight 549, and images were obtained using a Zeiss LSM 510 confocal microscope with Zen imaging software (Carl Zeiss, Inc., Thornwood, N.Y., USA). Transmission electron microscopy of LVS-infected human neutrophils was performed as we have previously described [15].

\section{Statistical Analysis}

Statistical comparisons were performed using one-way ANOVA followed by Tukey's multiple-comparisons post hoc test. All analyses utilized GraphPad Prism software v6.0. p values $<0.05$ were considered statistically significant.

\section{Results}

\section{F. tularensis Markedly Delays Constitutive Neutrophil Apoptosis}

Our published data demonstrate that $F$. tularensis strains, including LVS, profoundly prolong the lifespan of human neutrophils [17]. These studies utilized bacteria cultivated on $\mathrm{CHAB}$, and we show that similar results were obtained using LVS that was grown to mid-log phase with shaking in Brain Heart Infusion broth (fig. 1). Specifically, the data indicate that LVS infection markedly impairs constitutive PMN apoptosis, as indicated by An- 


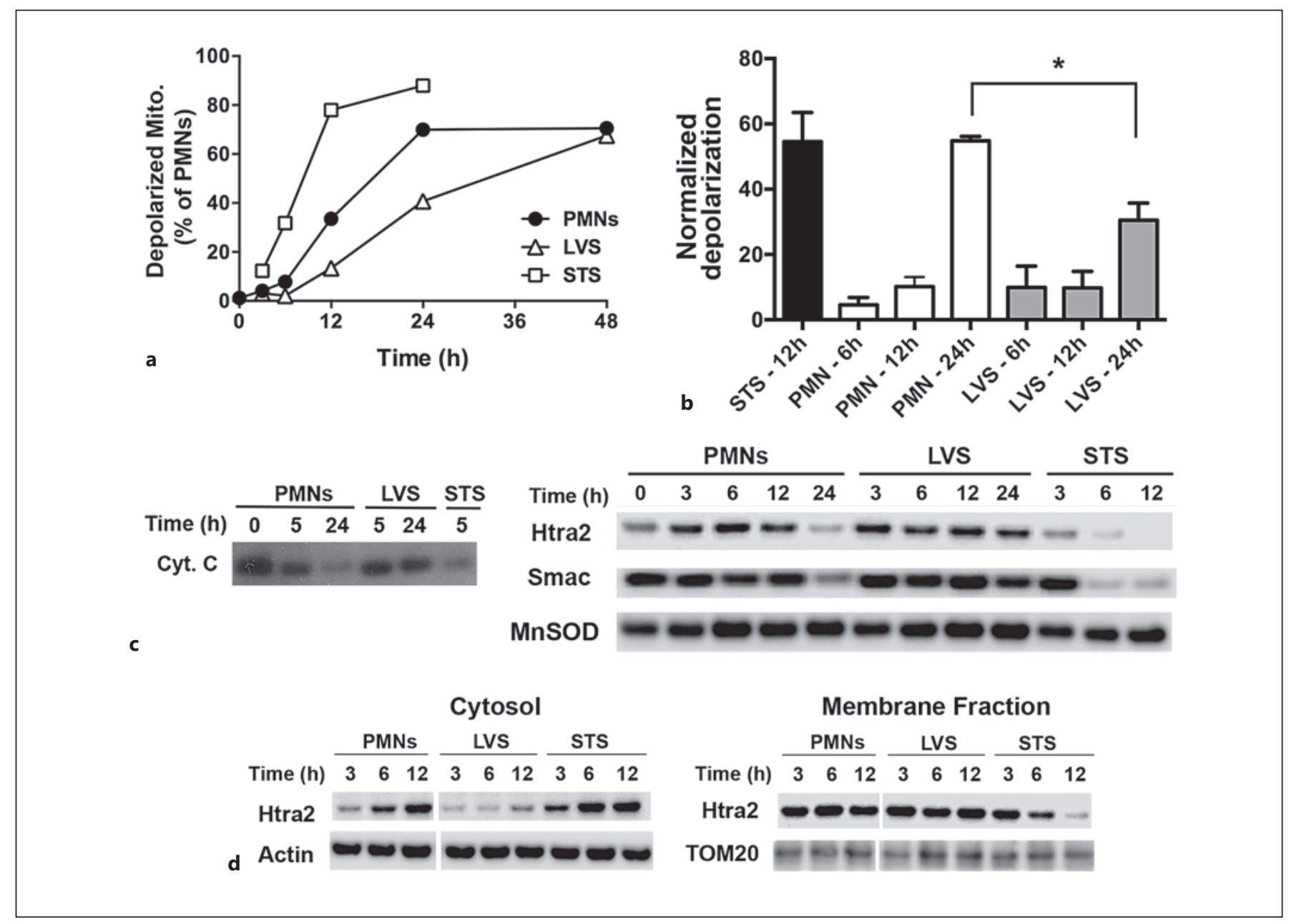

Fig. 2. F. tularensis sustains neutrophil mitochondrial integrity. PMNs were left untreated, infected with LVS or treated with STS as indicated. a, b Loss of mitochondrial membrane potential was quantified by JC-1 staining. a A representative time course of mitochondrial depolarization. Mito = mitochondria. $\mathbf{b}$ Pooled data normalized to the fluorescence at time zero, showing the mean \pm SEM of 3 independent experiments. ${ }^{*} \mathrm{p}<0.05$. c, d Outer mitochondrial membrane permeabilization was assessed by subcellular fractionation. c Immunoblots of cytochrome $c$ (Cyt. C), Htra2 and Smac retained in mitochondria-containing fractions at each time point and are representative of at least 4 independent experiments. d Matched immunoblots of cytosol and mitochondria-containing membrane fractions show delayed release of $\mathrm{Htra} 2$ into the cytosol during LVS infection and are representative of at least 4 independent experiments. $\beta$-actin, MnSOD and TOM20 were used as loading controls. nexin V-FITC staining which detects phosphatidylserine in the outer leaflet of the plasma membrane, and PI which detects cells that progress to late apoptosis or secondary necrosis (fig. 1a, b). In keeping with this, caspase- 3 activation was diminished and delayed (fig. 1c). Conversely, stimulation of the intrinsic apoptosis pathway using STS triggered rapid caspase- 3 activation that was apparent within $5 \mathrm{~h}$ (fig. 1c), in agreement with published data [17]. These results underscore the ability of external stimuli, including bacterial pathogens, to modulate PMN apoptosis and lifespan.

F. tularensis Inhibits Neutrophil Apoptosis

\section{Mitochondrial Integrity Is Sustained by F. tularensis Infection}

Mitochondria are major regulators of apoptosis, and although these organelles are less abundant in PMNs than in other cell types [19], they are readily apparent on transmission electron microscopy (online suppl. fig. 2). In healthy cells, mitochondria are intact and maintain a membrane potential $(\Delta \Psi \mathrm{m})$ across the outer mitochondrial membrane (OMM) that can be quantified using the fluorescent dye, JC-1 [28]. Disruption of the OMM is a very early event in constitutive PMN apoptosis that is re- 
quired for activation of caspase-9 [4, 5, 28]. As caspase-9 activation is significantly diminished and delayed by $F$. tularensis [17], we predicted that mitochondrial integrity might be sustained. To test this hypothesis, we used JC-1 and flow cytometry to quantify the loss of $\Delta \Psi \mathrm{m}$ over time. In each case, control (untreated) and LVS-infected PMNs were directly compared, and cells treated with $1 \mu \mathrm{M}$ STS were used as an additional positive control. In keeping with its ability to trigger caspase activation and cell death, STS caused rapid mitochondrial depolarization that was apparent in $80 \%$ of the PMNs by $12 \mathrm{~h}$ (fig. $2 \mathrm{a}$ ), which represented a 55 -fold change in JC-1 fluorescence relative to baseline measurements at time zero (fig. $2 \mathrm{~b}$ ). $\Delta \Psi \mathrm{m}$ declined more slowly in the control PMNs, and this was reduced further by LVS infection (fig. 2a). As differences between control and LVS-infected PMNs were statistically significant at $24 \mathrm{~h}$ (fig. $2 \mathrm{~b}$ ), our data indicate that F. tularensis infection preserved $\Delta \Psi \mathrm{m}$.

A second consequence of OMM permeabilization is the release of proapoptotic intermembrane space (IMS) proteins (cytochrome $c$, Smac and Htra2) into the cytosol. In this locale, cytochrome $c$ triggers formation of the apoptosome, which is a platform for the activation of caspase-9 $[4,18]$. We used subcellular fractionation and immunoblotting to further assess the effects of LVS on mitochondrial integrity. We used an established fractionation protocol [26] that was validated in our hands using antibodies to mitochondrial (MnSOD or TOM20) and cytosolic ( $\beta$-actin) proteins (fig. $2 \mathrm{c}, \mathrm{d}$, and data not shown). By this assay, cytochrome $c$ disappeared from mitochondria by $5 \mathrm{~h}$ after treatment with STS and by $24 \mathrm{~h}$ in the control cells undergoing constitutive apoptosis, but was retained in these organelles for at least $24 \mathrm{~h}$ following LVS infection (fig. 2c, left panel). Although cytochrome $c$ is essential for apoptosis, it is present at low levels in PMNs and can be difficult to detect $[18,28]$. We therefore extended our analyses to include Smac and Htra2, and obtained similar results (fig. 2c, right panels). Direct comparisons of matched cytosol and mitochondria-containing membrane fractions confirmed that Htra2 release from the mitochondrial IMS was accelerated by STS but diminished and delayed by LVS infection (fig. 2d; the densitometry of these and other immunoblots is shown in online suppl. fig. 1). Collectively, our data demonstrate that mitochondrial integrity is significantly prolonged by $F$. tularensis.

\section{Bax Translocation to Mitochondria Is Impaired}

Mitochondrial integrity is regulated by members of the Bcl-2 protein family and, among these, Bax, Bid, Bak,
Mcl-1 and BCL2A1 (formerly called Bfl-1) are expressed in PMNs $[4,18,29]$. During apoptosis, Bax translocates from the cytosol to mitochondria, oligomerizes alone or together with Bak, and inserts into the OMM, thereby forming pores that mediate IMS protein release [4, 5, 29]. We used confocal microscopy and subcellular fractionation to determine if LVS influenced Bax localization as a means to sustain mitochondrial integrity. In freshly isolated, healthy neutrophils, Bax is distributed throughout the cytosol [29], and appears as diffuse, very dim, red fluorescence (fig. 3a, asterisks). As these cells aged in culture and progressed to apoptosis, Bax translocated to mitochondria and accumulated on these organelles, as indicated by foci of bright red fluorescence (fig. 3a, arrows). Double staining with antibodies to MnSOD confirmed that the Bax-enriched structures that we observed were mitochondria (fig. 3b). Specifically, Bax translocation was apparent in a small subset of control PMNs by $8 \mathrm{~h}$ and increased progressively thereafter, such that $73 \pm 15 \%$ of cells contained bright Bax foci by $24 \mathrm{~h}$ (fig. 3c). As expected, Bax translocation was significantly accelerated in PMNs that were subjected to Fas-crosslinking or treated with STS to induce apoptosis (fig. 3a-c). Conversely, Bax translocation was significantly diminished and delayed by LVS. Thus, mitochondria positive for Bax were apparent in only $24 \pm 12 \%$ of the infected PMNs at 24 h postinfection (hpi; $\mathrm{p}<0.001$ vs. controls), and this increased to only $39 \pm 8 \%$ of PMNs by 48 hpi (fig. $3 \mathrm{a}-\mathrm{c}$ ).

In additional experiments, we used immunoblotting to define the time course of Bax disappearance from PMN cytosol (fig. 3d). By this assay, Bax in the control neutrophil cytosol was diminished slightly at 6 and $12 \mathrm{~h}$, and was absent by $24 \mathrm{~h}$. As expected, Bax disappeared more rapidly from PMN cytosol following Fas-crosslinking or STS treatment, and was absent or nearly undetectable by $12 \mathrm{~h}$. On the other hand, cytosolic Bax remained unchanged until at least $12 \mathrm{~h}$ after LVS infection, and was only partially diminished by 24 hpi. Collectively, our microscopy and subcellular fractionation data demonstrate that $F$. $t u$ larensis disrupts Bax translocation to the mitochondria and, as such, provide an insight into mechanisms that mediate enhanced mitochondrial integrity.

\section{Bid Processing Is Diminished, but Mcl-1 and BCL2A1} Are Unchanged

Caspase- 8 is the initiator caspase of the extrinsic pathway and is also activated during constitutive PMN apoptosis [5]. In neutrophils, the extrinsic pathway is not sufficient for cell death without additional signal amplification via the intrinsic pathway [4]. A critical link 


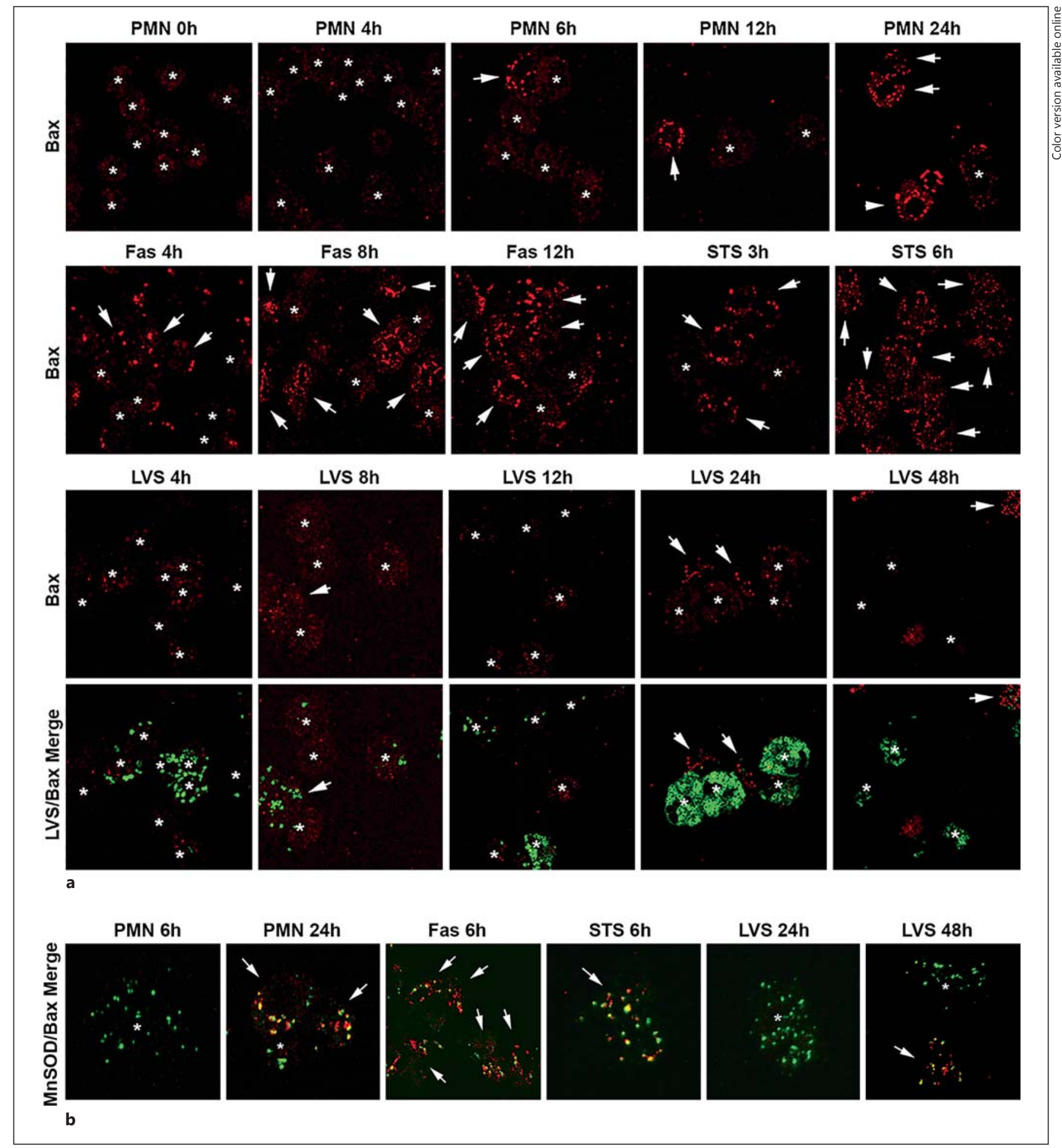

Fig. 3. F. tularensis inhibits Bax translocation to mitochondria. Neutrophils were left untreated, infected with LVS or were treated with Fas-crosslinking antibodies or STS as indicated. a Bax translocation to and accumulation on neutrophil mitochondria were assessed at the indicated time points by confocal microscopy. Representative images show Bax (red) and LVS (green). Arrows and asterisks indicate PMNs that do and do not show mitochondrial Bax accumulation, respectively. b Merged confocal images show the extent of Bax (red) colocalization with the mitochondrial marker MnSOD (green). Arrows and asterisks indicate PMNs that do and do not show mitochondrial Bax accumulation, respectively. Colors refer to the online version only. 


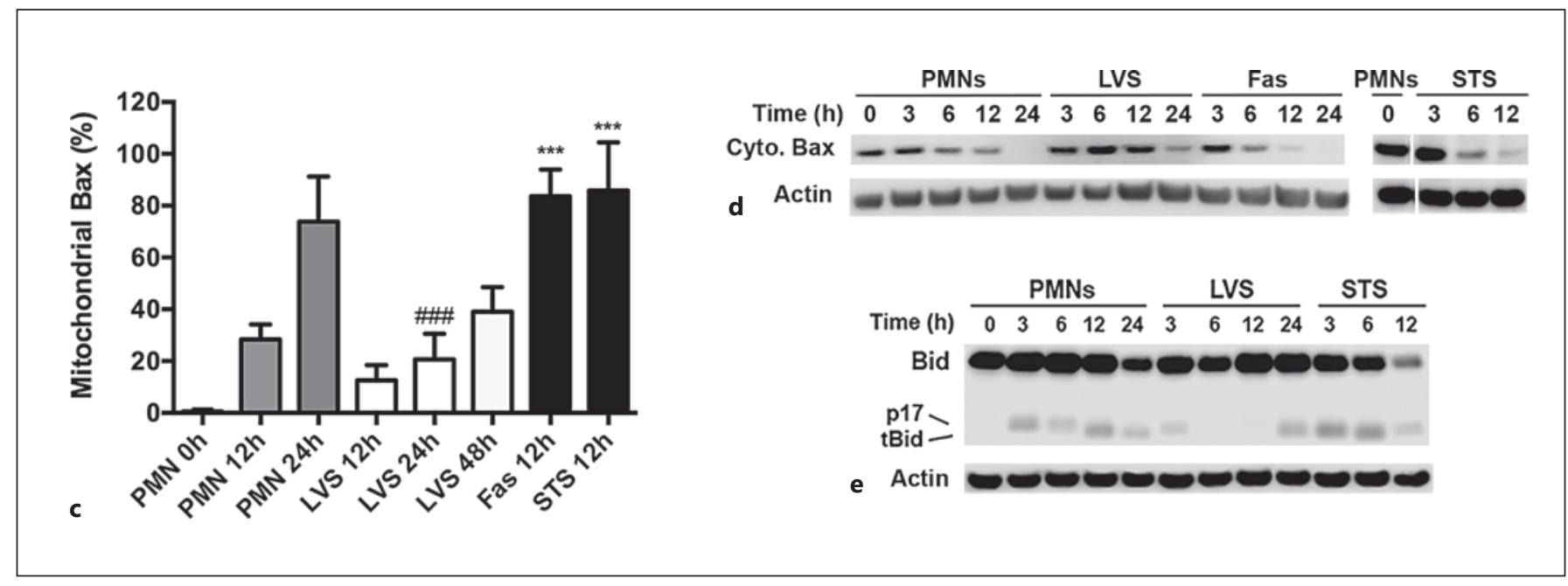

Fig. 3. F. tularensis inhibits Bax translocation to mitochondria. Neutrophils were left untreated, infected with LVS or were treated with Fas-crosslinking antibodies or STS as indicated. c Pooled microscopy data indicate the kinetics of Bax translocation. Data are the mean \pm SD from 8 independent experiments. ${ }^{* * *} \mathrm{p}<0.001$ vs. control and LVS-infected PMNs at $12 \mathrm{~h}$. ${ }^{\# \#} \mathrm{p}<0.001$ vs. control PMNs at 24 h. d Immunoblots indicate the kinetics of Bax disap-

between these pathways is $\mathrm{Bid}$, a prodeath $\mathrm{Bcl}-2$ family protein. Caspase- 8 cleaves Bid to its truncated active form (tBid; $15 \mathrm{kDa}$ ), which facilitates the recruitment and insertion of Bax into OMMs and also contributes directly to Bax activation $[4,29]$. We have shown that caspase- 8 processing is nearly ablated by LVS [17], but possible effects on Bid are unknown. We therefore used immunoblotting to examine the conversion of Bid to tBid. With this assay, full-length $\mathrm{Bid}(22 \mathrm{kDa})$ was abundant in PMNs (fig. 3e), and small amounts of a p17 cleavage product that is generated in healthy cells by an unknown mechanism [30] were detected in untreated PMNs at 3 and $6 \mathrm{~h}$ (fig. 3e). tBid was detected at 12 and $24 \mathrm{~h}$, and at the latter time point, full-length Bid was also diminished. Following STS treatment, the rate and extent of Bid processing was accelerated, such that tBid was present by $3 \mathrm{~h}$ and Bid had declined sharply by $12 \mathrm{~h}$ (fig. 3e). In marked contrast, Bid was not depleted following LVS infection and tBid was not detected until $24 \mathrm{hpi}$. These data indicate that Bid processing was impaired. Of note, the p17 Bid fragment also disappeared from the LVS-infected PMNs at time points $>3 \mathrm{hpi}$, but as the function of p17 Bid is unknown, the possible significance of this finding remains to be determined. pearance from PMN cytosol. Data are representative of at least 3 independent experiments. e Immunoblots show the extent of Bid $(22 \mathrm{kDa})$ processing to its proapoptotic truncated (tBid) $15-\mathrm{kDa}$ form or a p17-kDa form of unknown function. Data are representative of 3 independent experiments. $\beta$-actin was used as the loading control.

Mcl-1 and BCL2A1 are antiapoptotic members of the Bcl-2 family that play an important role in neutrophil survival by binding to Bax and Bak and preventing their activation $[2,4,18]$. Certain cytokines extend PMN lifespan by upregulating BCL2A1 or Mcl-1 $[2,4,19]$. Our microarray and qRT-PCR data indicate that MCL1 is downregulated 2-fold by LVS whereas BCL2A1 mRNA increases 4 -fold at $24 \mathrm{hpi}$ [20]. Nonetheless, we show here that LVS infection had no apparent effect on the encoded proteins, as both appeared unchanged relative to the untreated controls (fig. 4a, b).

\section{Calpastatin-Calpain Ratios Are Elevated in Infected PMNs}

Downstream of the mitochondria, apoptosis is modulated by the relative abundance of pro- and antiapoptotic regulatory factors in the cytosol. XIAP is the most potent antiapoptotic protein at this locale, and sustains cell survival by directly binding to and inhibiting the processing and activation of caspase- 9 and caspase-3 [19, 31]. During apoptosis, XIAP is degraded to an inactive p30 fragment by the cysteine protease calpain [32]. However, as we demonstrated previously [20] and show again here (fig. 5a), XIAP degradation is markedly impaired by LVS, and full-length XIAP remains abundant until at least 48 


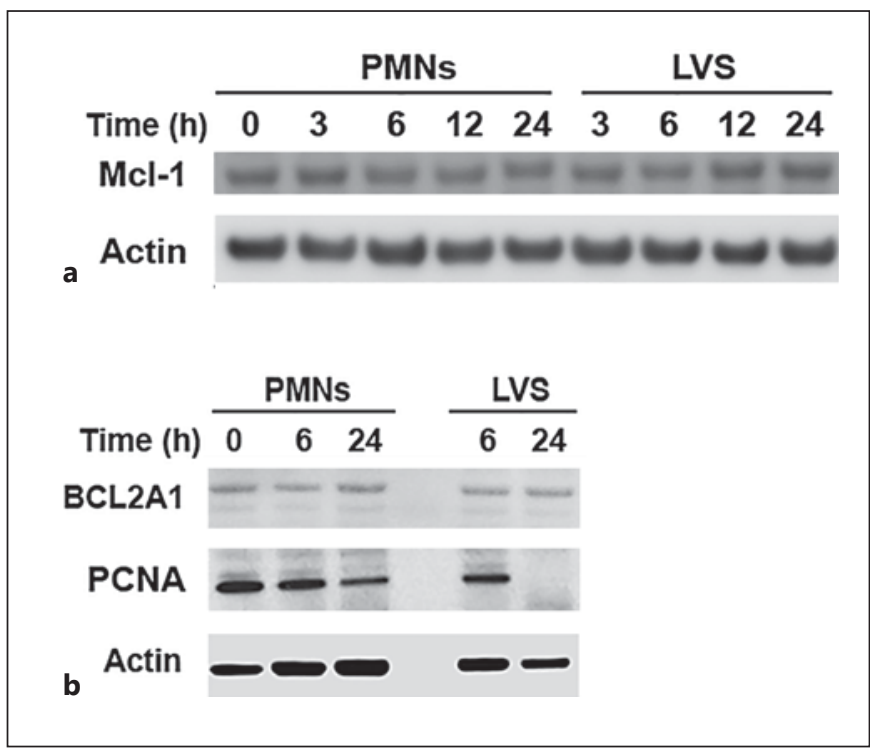

Fig. 4. Effects of F. tularensis on Mcl-1, BCL2A1 and PCNA. Neutrophils were left untreated, were infected with F. tularensis or were treated with STS for the indicated amounts of time. Immunoblots of cell lysates were probed to detect the indicated apoptosis regulatory factors and $\beta$-actin was used at the loading control. a Mcl- 1 and $\beta$-actin. b BLC2A1, PCNA and $\beta$-actin. In each case, the immunoblots are representative of at least 3 independent experiments.

hpi, but how this is achieved is unclear. In healthy cells, the activity of calpain is blocked by a direct association with its endogenous inhibitor, calpastatin [32-34]. Thus, downregulation of calpastatin during apoptosis allows degradation of calpain substrates including XIAP [32]. $C A S T$, which encodes calpastatin, is significantly upregulated in LVS-infected PMNs at both 12 and 24 hpi [20]. As increased calpastatin/calpain ratios can stabilize XIAP and impair PMN death [33], we assessed the relative abundance of calpain and calpastatin in our system. Our immunoblotting data confirm that multiple calpastatin isoforms are expressed in PMNs [33], and demonstrate that the largest isoforms were selectively induced by LVS, whereas calpain levels declined slightly or appeared unchanged (fig. $5 \mathrm{~b}$ and data not shown). Consistent with the finding of elevated calpastatin/calpain ratios, total calpain activity in LVS-infected PMNs at 24 hpi increased by only about $25 \%$ relative to baseline (fresh PMNs), and as such was significantly reduced relative to the aged, uninfected controls and PMNs treated with STS (fig. 5c). These data extend our previous studies on CAST expression, and provide additional evidence to suggest that LVS modulates the calpastatin-calpain-XIAP-caspase axis.

F. tularensis Inhibits Neutrophil Apoptosis

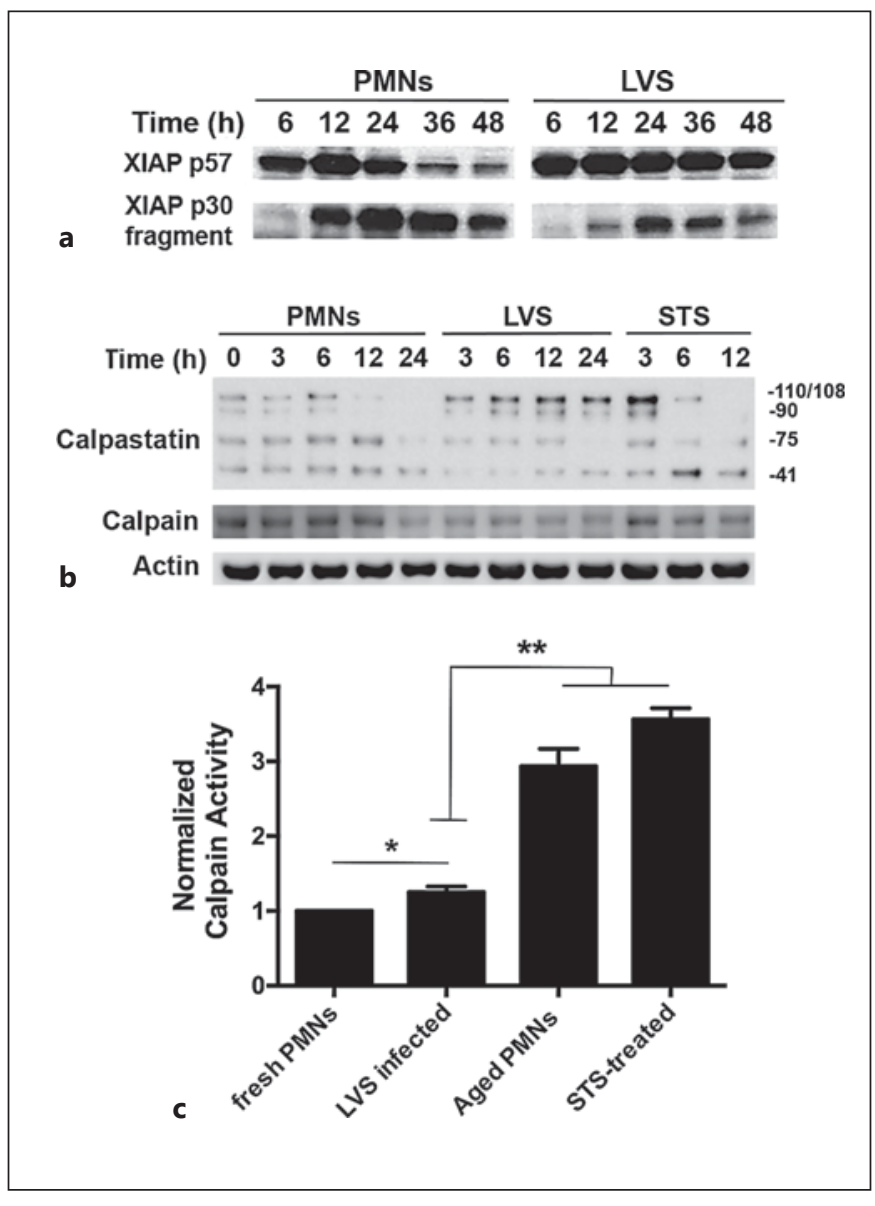

Fig. 5. Effects of F. tularensis on XIAP, calpastatin and calpain. Neutrophils were left untreated, were infected with $F$. tularensis or were treated with STS as indicated. Immunoblots of cell lysates were probed to detect apoptosis regulatory factors or $\beta$-actin, and calpain activity was measured in whole cells using a fluorogenic substrate. a LVS infection impairs XIAP degradation by calpain. b LVS differentially influences calpain and calpastatin isoforms in neutrophils. In each case, the immunoblots are representative of $\geq 3$ independent determinations. c Relative calpain activity in freshly isolated PMNs compared with cells that were treated with STS for $12 \mathrm{~h}$ or incubated for $24 \mathrm{~h}$ in the presence and absence of LVS. Data are the mean \pm SD of 3 independent experiments. ${ }^{*} \mathrm{p}<$ 0.05 ; $^{* *} \mathrm{p}<0.01$.

PCNA Disappears from F. tularensis-Infected Cells

PCNA is an additional regulatory factor that also contributes to caspase inhibition in PMNs $[4,18,35]$. Our previous studies identified $P C N A$ as a gene that was progressively downregulated by LVS over 6-24 hpi [20]. In agreement with this, our current results indicate that PCNA disappeared more rapidly from LVS-infected PMNs at time points $>6$ hpi (fig. 4b). Additional experiments confirmed these results and showed that PCNA remained un- 

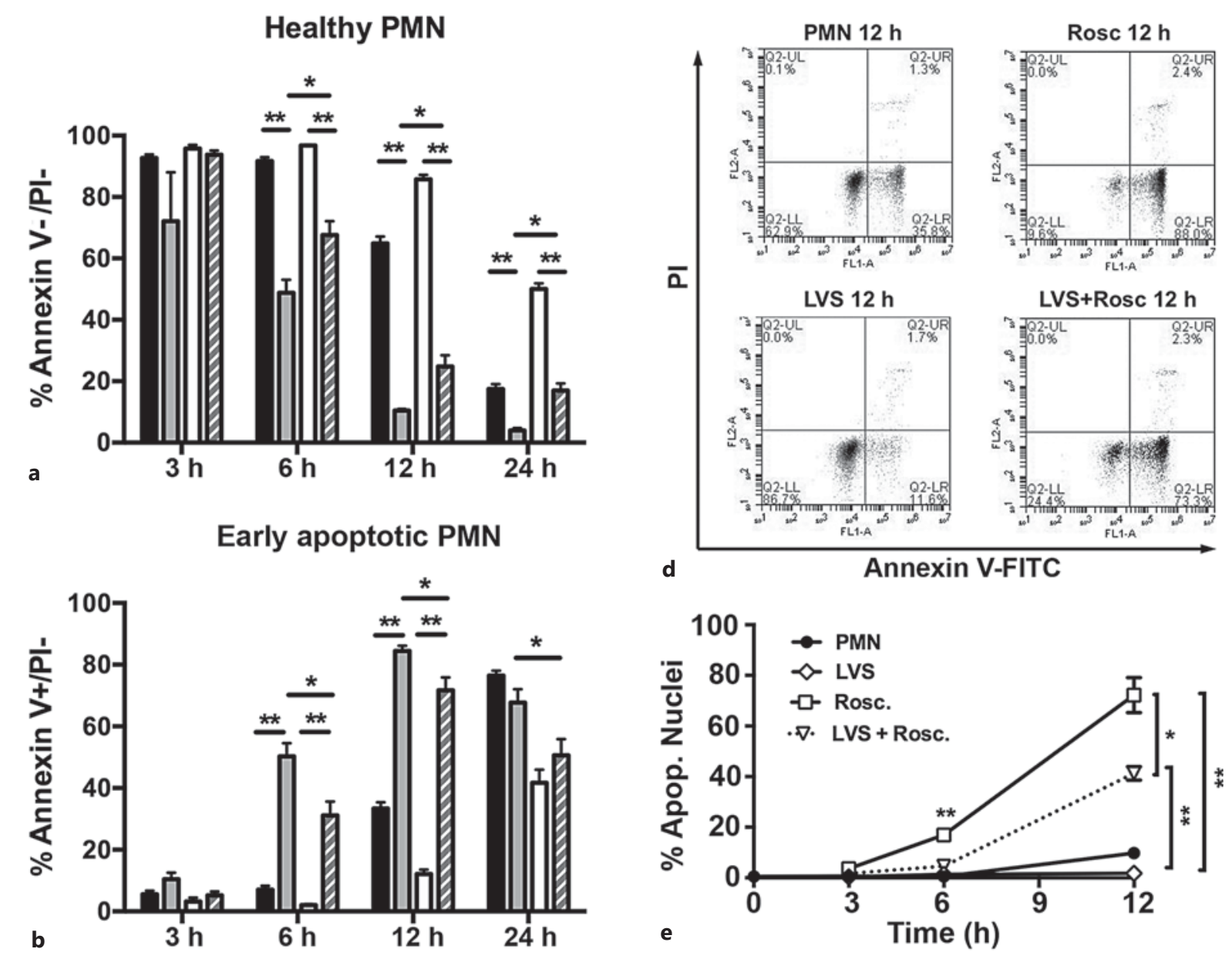

Late apoptotic/necrotic PMN
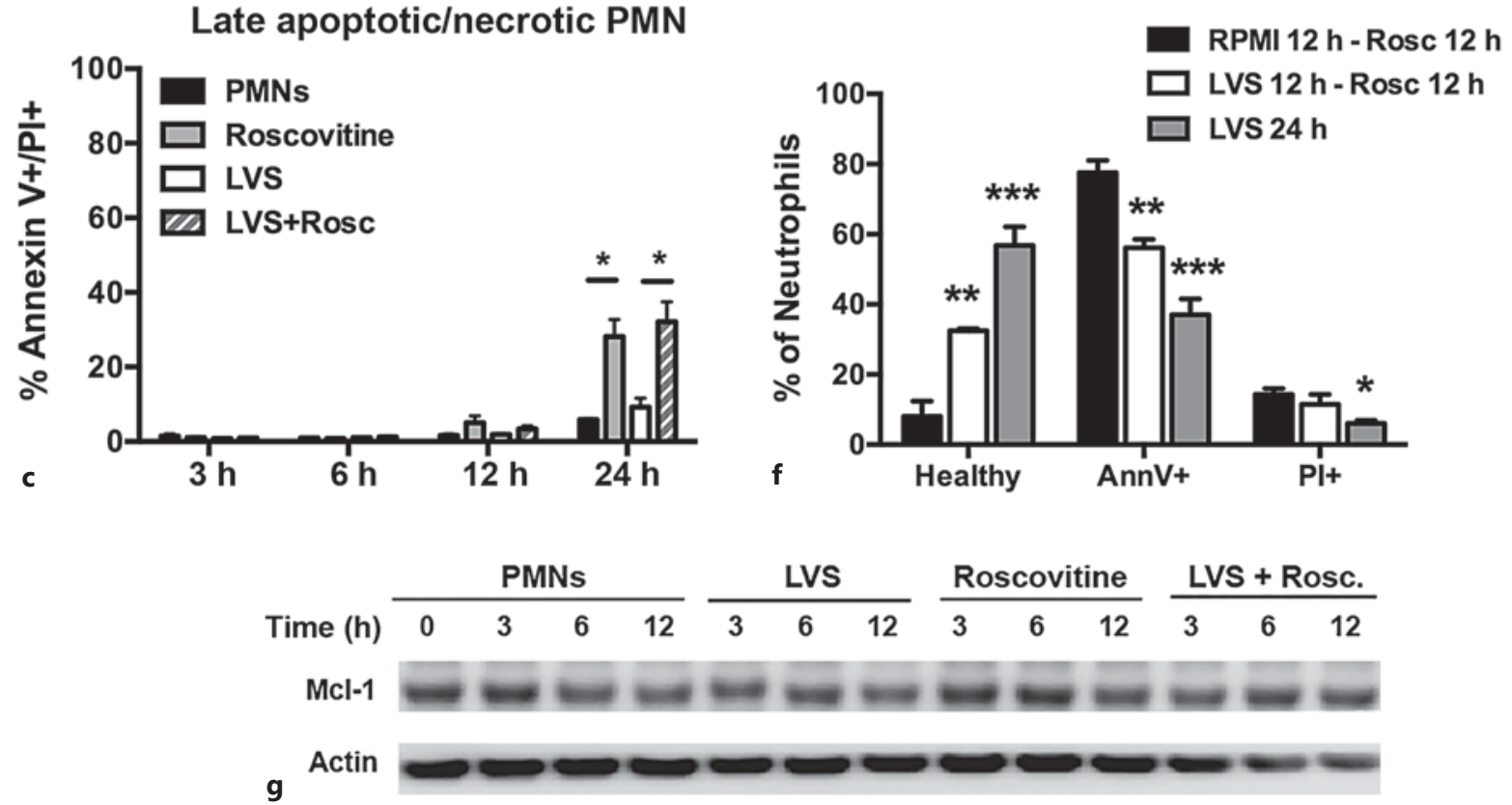

(For legend see next page.) 
changed in control PMNs over $18 \mathrm{~h}$, but was diminished in LVS-infected cells by 12 hpi and was absent or nearly absent at $18 \mathrm{hpi}$ (data not shown). We therefore conclude that PCNA is not essential for neutrophil survival and delayed apoptosis during $F$. tularensis infection.

\section{F. tularensis-Infected Neutrophils Resist \\ $R$-Roscovitine-Induced Apoptosis}

There is significant interest in the development of apoptosis-inducing drugs as primary or adjunctive therapeutics to treat inflammatory and infectious disorders that are characterized by profound PMN accumulation and tissue destruction [4]. One agent in this class is the cyclin-dependent kinase (CDK) inhibitor R-roscovitine, which induces PMN apoptosis in a dose- and time-dependent manner and can negate the prosurvival effects of GM-CSF $[4,21]$. The effects of R-roscovitine on F. tularensis infection are unknown [14]. Thus, we first determined that $20 \mu \mathrm{M}$ R-roscovitine was not toxic to LVS and had no effect on bacterial viability or growth rate in broth (data not shown). Next, we directly compared the ability of R-roscovitine to induce apoptosis of control and LVSinfected PMNs. R-roscovitine was added $1 \mathrm{~h}$ after the initiation of infection or incubation at $37^{\circ} \mathrm{C}$, and the progression of cells to apoptosis was assessed at multiple time points over $24 \mathrm{~h}$ by Annexin V-FITC/PI staining and by the analysis of nuclear morphology (fig. 6a-e). The effects of R-roscovitine on human neutrophil viability were apparent by $3 \mathrm{~h}$ (the earliest time point examined), but were not statistically significant at this stage (fig. 6a, b). By $6 \mathrm{~h}$, $50 \pm 10 \%$ of R-roscovitine-treated PMNs were apoptotic, as judged by Annexin $\mathrm{V}$ staining, and this increased to 84 $\pm 4 \%$ by $12 \mathrm{~h}$ compared with only $7 \pm 3 \%$ and $33 \pm 4 \%$ of the respective, untreated controls (fig. 6b). In addition, 28 $\pm 10 \%$ of the R-roscovitine treated cells were PI+ by $24 \mathrm{~h}$, indicating a progression to late apoptosis or secondary necrosis (fig. 6c).

Fig. 6. F. tularensis attenuates the ability of R-roscovitine to induce neutrophil apoptosis. Effects of R-roscovitine on apoptosis of control and LVS-infected PMNs were quantified by Annexin V-FITC/ PI staining or by analysis of nuclear morphology. a-e, $\mathbf{g}$ R-roscovitine was added $1 \mathrm{~h}$ after LVS infection. a-c Graphs show the percentage of PMNs at each time point and condition that are healthy (Annexin V-/PI-), in early apoptosis (Annexin V+/PI-), or have progressed to late apoptosis/secondary necrosis (Annexin $\mathrm{V}+$ / $\mathrm{PI}+$ ), respectively. Data are the mean $\pm \mathrm{SD}$ of 5 independent experiments. ${ }^{* *} \mathrm{p}<0.001 ;{ }^{*} \mathrm{p}<0.05$. d Representative flow cytometry plots at the 12-hour time point. e Percentage of PMNs with an apoptotic nuclear morphology. Data are the mean \pm SD of 3 inde-
Although R-roscovitine also enhanced the apoptosis of the F. tularensis-infected PMNs at 6 and $12 \mathrm{~h}$, the effects of this drug were significantly blunted by LVS (fig. 6a, b). These differences are perhaps most easily discerned by the comparison of the representative flow plots (fig. 6d), and these results were independently confirmed by analysis of PMN nuclear morphology (fig. 6e). We next asked if extending the duration of LVS infection prior to drug treatment influenced the outcome. To this end, we left PMNs in medium alone or infected them with LVS for $12 \mathrm{~h}$ prior to treatment with R-roscovitine for an additional $12 \mathrm{~h}$. The data in figure $6 \mathrm{f}$ confirm the ability of LVS to significantly inhibit R-roscovitine-induced apoptosis (78 \pm 6 vs. $56 \pm 4 \%$ Annexin V+ cells; $\left.{ }^{* *} \mathrm{p}<0.01\right)$ and, under these conditions, the proportion of PMNs that remained healthy increased 4 -fold ( $32 \pm 1$ vs. $8 \pm 7 \%$; $p<0.01$ ) compared with only 2.5 -fold when using the 1 -hour preinfection protocol ( $25 \pm 8$ vs. $10 \pm 1 \%$; $\mathrm{p}<0.05$; fig. $6 \mathrm{a}$ ). Nevertheless, our findings also indicate that apoptosis remained significantly accelerated relative to PMNs that were infected with LVS in the absence of drug treatment (fig. 6f). Our data therefore confirm the ability of R-roscovitine to induce apoptosis, and demonstrate that its efficacy is significantly diminished but not ablated by $F$. tularensis.

\section{Mcl-1 Is Not Downregulated by R-Roscovitine}

How CDK inhibition leads to PMN apoptosis induction is unclear. RNA polymerase II is a CDK substrate that can influence the expression of MCL1, and R-roscovitine treatment correlates with $M C L 1$ downregulation in some studies [21, 22]. Nonetheless, in our system, the amount of Mcl-1 protein in PMN lysates was not appreciably altered during the first $12 \mathrm{~h}$ after R-roscovitine treatment (fig. $6 \mathrm{~g}$ ), despite robust apoptosis induction (fig. 6b). We therefore conclude that, under the conditions used here, Mcl-1 downregulation is not essential for R-roscovitine-triggered PMN death.

pendent experiments. ${ }^{*} \mathrm{p}<0.05 ;{ }^{* *} \mathrm{p}<0.01$. f PMNs were left in medium or infected with LVS for $12 \mathrm{~h}$ prior to treatment with Rroscovitine for $12 \mathrm{~h}$, or were infected with LVS for $24 \mathrm{~h}$ in the absence of R-roscovitine. Annexin V-FITC/PI staining results are the mean \pm SD of 3 independent experiments. ${ }^{* *} \mathrm{p}<0.01$ for LVS at $12 \mathrm{~h}-\mathrm{R}$-roscovitine at $12 \mathrm{~h}$ versus both other treatments. ${ }^{* * *} \mathrm{p}<$ $0.001 ;{ }^{*} \mathrm{p}<0.05$ for LVS at $24 \mathrm{~h}$ vs. RPMI at $12 \mathrm{~h}-\mathrm{R}$-roscovitine at $12 \mathrm{~h}$. $\mathbf{g}$ Immunoblots of PMN lysates prepared at the indicated time points were probed to detect Mcl-1 with $\beta$-actin as the loading control. Data are from 1 experiment representative of 3 independent determinations. Rosc. $=$ R-roscovitine. 


\section{Discussion}

Neutrophils are important regulators of the inflammatory response, and timely apoptosis of these cells at sites of infection permits the safe removal of effete cells and their infectious cargo. Additionally, this process minimizes tissue destruction and stimulates the reprogramming of macrophages to a proresolution phenotype. There is now extensive evidence to demonstrate that dysregulation of these events contributes to the pathogenesis of many infectious and inflammatory disorders $[2,6,14]$. During pneumonic tularemia, neutrophil accumulation, pyogranuloma formation and tissue necrosis together contribute to disease lethality. Macrophages and neutrophils play distinct roles in pathogenesis, as macrophages are major vehicles for bacterial growth and dissemination whereas PMNs are critical drivers of an ineffective and damaging inflammatory response $[13,14]$. Consistent with this model, we previously demonstrated that $F$. tularensis inhibits human neutrophil apoptosis, as indicated by defects in caspase activation that correlate with diminished and delayed phosphatidylserine externalization, nuclear condensation and DNA fragmentation as well as changes in gene expression $[17,20]$. The results of this study provide insight into the underlying molecular mechanisms of apoptosis inhibition and are significant for several reasons. First, we demonstrate that $F$. tularensis sustains mitochondrial integrity via its effects on Bax translocation and Bid processing. Second, we show that PCNA disappears rapidly from $F$. tularensis-infected cells, hereby demonstrating that this protein is not essential for PMN survival. Third, we demonstrate that $F$. tularensis can undermine the efficacy of R-roscovitine, an apoptosis-inducing drug that is currently in clinical trials.

Mitochondria are central regulators of PMN apoptosis, and it has been established that mitochondrial integrity is essential for cell survival, whereas OMM disruption and subsequent cytochrome $c$ release can commit cells to death by stimulating apoptosome formation and caspase- 9 activation $[4,29,32]$. Mitochondrial integrity is governed by the opposing actions of $\mathrm{Bcl}-2$ family proteins, and $\mathrm{Bax}$ is of particular interest as it is directly responsible for pore formation in the OMM. Among the earliest events in constitutive PMN apoptosis is the translocation of Bax from the cytosol to the mitochondria $[4,29]$. The results of this study demonstrate that Bax translocation is significantly diminished and delayed by LVS to $48 \mathrm{hpi}$, and mitochondrial integrity and $\Delta \Psi \mathrm{m}$ are sustained (fig. 2,3 ). Inhibition of Bax translocation likely accounts in large part for the defect in caspase- 9 activation that we reported previously [17]. Our current and published findings suggest that $F$. tularensis acts at multiple points to disrupt Bax localization and function. In particular, we have shown that $B A X$ mRNA is profoundly downregulated during LVS infection, and that the total amount of Bax in PMN lysates declines slightly at 12-24 hpi, in keeping with the long halflife of this protein $[4,20]$. In addition, Mcl-1 sequesters Bax in the cytosol of healthy cells, and as such upregulation of MCL1 contributes to the ability of growth factors such as G-CSF and GM-CSF to extend PMN lifespan [4, 36, 37]. Although LVS has no significant effect on Mcl-1 protein (fig. 4a) and slightly decreases MCL1 expression [20], this bacterium could potentially enhance cytosolic sequestration of Bax via other mechanisms that involve phosphorylation of Mcl-1 and/or Bax, or utilize the related prosurvival protein BCL2A1 $[36,38,39]$. Effects on phosphorylation of Mcl-1 are of particular interest, as CDK1, CDK2 or JNK-mediated phosphorylation are required for its heterodimerization with the proapoptotic Bcl-2 family proteins [36], and our data suggest that CDK signaling may be prolonged in LVS-infected cells, as indicated by upregulation of CDK2 and CDK7 [20] and enhanced resistance to R-roscovitine (fig. 6, discussed below). During apoptosis, the effects of Mcl- 1 are counteracted by tBid, which stimulates Bax translocation to mitochondria and induces conformational changes that are required for Bax activation, oligomerization and OMM insertion $[4,29,40]$. We show here that conversion of Bid into tBid is impaired (fig. 3e), thereby defining another mechanism of Bax interference and mitochondrial stabilization during LVS infection. At the molecular level, conversion of Bid to its active, truncated form is mediated by caspase- 8 [29], and we have shown that caspase- 8 activity is nearly abolished by $F$. $t u$ larensis infection [17]. Notably, caspase- 8 activation during constitutive PMN apoptosis can occur in the absence of death-receptor signaling and be initiated instead by reactive oxygen species or other mechanisms that disrupt azurophilic granule integrity, leading to procaspase- 8 activation by liberated cathepsins, which may also contribute to activation of Bid [19]. However, a distinguishing feature of $F$. tularensis is its ability to induce global inhibition of the NADPH oxidase complex throughout infected PMNs $[15,16]$, which acts in concert with $B A X$ downregulation to prevent activation of the PICD pathway [20] and also accounts for the defects in Bid processing and caspase- 8 activation reported here and in previous work (fig. 3e) [17].

The mechanisms that operate downstream of mitochondria to regulate PMN apoptosis are also complex and, at a minimum, involve the regulatory factors XIAP, 


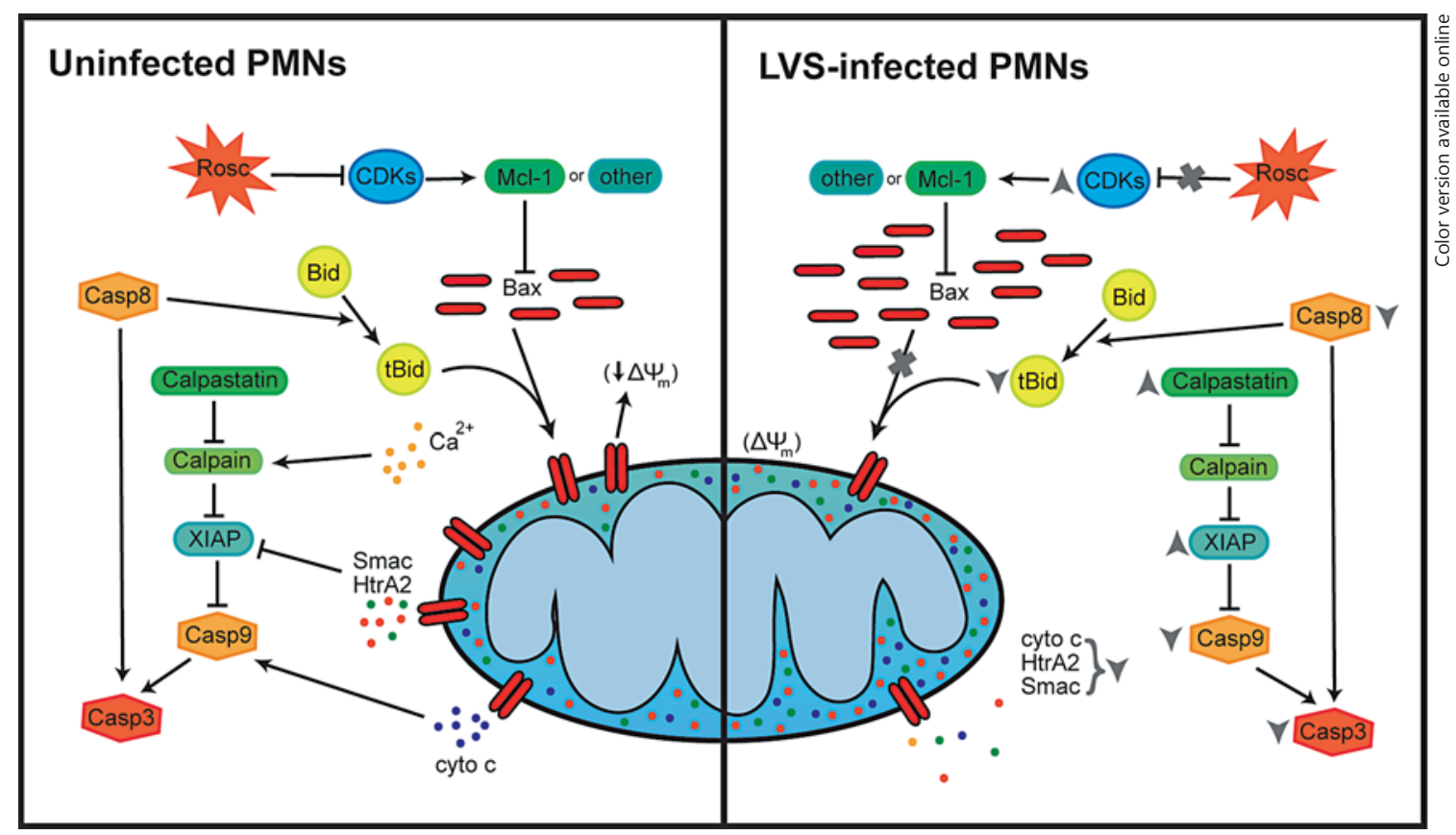

Fig. 7. Model summarizing the effects of $F$. tularensis on mitochondrial integrity and associated regulatory factors that act upstream and downstream of mitochondria to enhance or curtail neutrophil apoptosis. cyto $\mathrm{c}=\mathrm{Cy}$ tochrome $c$.

calpain, calpastatin, PCNA, and p21Waf1. XIAP binds directly to caspase- 9 and caspase- 3 , and is the most potent caspase inhibitor in the cytosol [31]. During apoptosis, $\mathrm{XIAP}$ is degraded by calpain, a constitutively active protease that is constrained in healthy cells by calpastatin [32, $34,41]$. As full-length XIAP is sustained for up to $48 \mathrm{hpi}$, and generation of a calpain-derived p30 fragment is markedly diminished and delayed [20] (fig. 5a), we hypothesized that the calpastatin-calpain-XIAP axis contributes to the defect in the processing and activation of caspase- 9 and caspase- 3 that occurs in F. tularensis-infected PMNs [17]. Accordingly, CAST, which encodes calpastatin, is upregulated by LVS [20], and we show here that a subset of calpastatin isoforms is specifically enriched in infected neutrophils (fig. 5b), whereas calpain activity is significantly reduced (fig. 5c). As each molecule of calpastatin inhibits 4 molecules of calpain and an excess of calpastatin is sufficient to prevent apoptosis [41], our data suggest that this mechanism contributes to enhanced PMN longevity during F. tularensis infection. Nonetheless, these data do not exclude roles for other mechanisms that may also delay XIAP degradation in our system. For example, a direct role for mitochondrial in- tegrity is suggested by the fact that XIAP can also be degraded by the serine protease Htra2 [42].

PCNA is a scaffolding protein in PMN cytosol that impairs the activation of caspase- 9 and caspase- 3 by direct binding, and previous studies have proposed that PCNA is essential for neutrophil survival $[18,35]$. Nonetheless, we demonstrate that PCNA disappeared rapidly and selectively from LVS-infected PMNs and was undetectable or nearly absent by $18 \mathrm{hpi}$ (fig. $4 \mathrm{~b}$ and data not shown) despite sustained cell viability. As such, our data are the first to indicate that PCNA is not essential for neutrophil survival in all circumstances. Based on studies of a cellpermeable peptide, it has been proposed that PCNA can be displaced from caspase- 9 by $21 \mathrm{Waf} 1$, leading to PCNA degradation by the proteasome [35]. In agreement with this model and our current results, $P C N A$ expression progressively declines beginning at $6 \mathrm{hpi}$ with LVS, whereas expression of CDKN1A, which encodes $\mathrm{p} 21 \mathrm{Waf} 1$, progressively increases over 12-48 hpi [20]. Additional studies are clearly needed to better define the shared and distinct roles of XIAP, PCNA and their binding partners in PMNs. Nevertheless, our data underscore the complexity of neutrophil apoptosis signaling as well as the 
ability of bacterial pathogens to inform studies of fundamental biological processes.

Major challenges in pneumonic tularemia treatment are the nonspecific nature of the presenting symptoms and the short interval between the onset of symptoms and severe illness or death. For this reason, there is an interest in the development of treatments that enhance antibiotic efficacy [14]. Currently, in clinical trials, R-roscovitine is of interest as an apoptosis-inducing agent that reverses the prosurvival effects of GM-CSF, promotes the resolution of inflammatory disorders that are characterized by aberrant PMN accumulation including carrageenan-induced pleurisy, bleomycin-induced lung injury and arthritis [21,22], and also synergizes with antibiotics for treatment of pneumococcal meningitis [43]. Here, we confirmed the ability of R-roscovitine to induce PMN apoptosis within $6 \mathrm{~h}$, but also discovered that its efficacy was significantly undermined by F. tularensis, particularly if drug treatment was delayed until $12 \mathrm{~h}$ after the initiation of infection (fig. 6). These data distinguish $F$. tularensis from other stimuli studied to date, and are also noteworthy as R-roscovitine inhibits CDKs 1, 2, 5, 7 and 9, which are present at low levels in mature PMNs $[22,44]$. As we have shown that $C D K 2$ and $C D K 7$ are significantly upregulated $12-48 \mathrm{hpi}$ with LVS [20], and this correlates with the kinetics of enhanced R-roscovitine resistance (fig. 6f), our data suggest a mechanism that merits further study.

It has also been proposed that R-roscovitine induces apoptosis via effects on MCL1, which is a downstream target of the CDK substrate RNA polymerase II [21, 22]. However, our data clearly demonstrate that loss of Mcl-1 is not essential for R-roscovitine-induced PMN death, necessitating consideration of other mechanisms and targets. For example, R-roscovitine could conceivably influence CDK-dependent Mcl-1 phosphorylation [36], and other relevant players likely await discovery as R-roscovitine significantly alters the PMN transcriptome including 268 genes linked to apoptosis [45]. Finally, it is also important to note that although there is abundant evidence that CDK inhibitors can induce apoptosis, how these kinases promote neutrophil survival is poorly defined. Moreover, p21Waf1, which is upregulated by LVS and may trigger PCNA degradation, is also an endogenous CDK inhibitor with specificity for CDKs 2, 4 and 6 [22]. Thus, it will be of interest in future studies to determine if the ability of $F$. tularensis to resist apoptosis in the face of PCNA depletion and R-roscovitine treatment are mechanistically linked, as, ultimately, it may be the balance between CDKs and their endogenous or exogenous inhibitors that governs PMN fate.

Altogether, the results of this study provide significant insights into the mechanisms of neutrophil apoptosis inhibition by F. tularensis, and support the integrated model shown in figure 7. In particular, we define a critical role for mitochondrial stabilization that correlates with the defects in Bax translocation and Bid processing. Our data also show that among the cytosolic regulatory factors, calpastatin and XIAP appear to be important for cell viability whereas PCNA is not. Regulation of apoptosis is complex, and our data reveal distinctive and potentially important features of $F$. tularensis, including its ability to deplete PCNA and undermine R-roscovitine efficacy, which provide a framework for future studies on PMN apoptosis, including CDK survival signaling.

\section{Acknowledgements}

This study was supported in part by a VA Merit Review Grant 5 I01 BX002108 and funds from the National Institutes of Health (NIAID R01 AI073835 and P01 AI044642) awarded to L.-A.H.A., and predoctoral fellowships awarded to J.M.M. and L.C.K. (NIAID T32 AI007511). We also acknowledge the use of the University of Iowa Central Microscopy Research Facility, which is supported in part by funds from the Carver College of Medicine.

\section{Disclosure Statement}

There are no conflicts of interest.
References
1 Nauseef WM: How human neutrophils kill and degrade microbes: an integrated view. Immunol Rev 2007;219:88-102.

2 Kennedy A, DeLeo F: Neutrophil apoptosis and the resolution of infection. Immunol Res 2009;43:25-61.

3 Gabelloni M, Trevani A, Sabatté J, Geffner J: Mechanisms regulating neutrophil survival and cell death. Semin Immunopathol 2013; 35:423-437.
4 McCracken JM, Allen L-AH: Regulation of human neutrophil apoptosis and lifespan in health and disease. J Cell Death 2014;7:15-23.

5 Kobayashi SD, DeLeo FR: Role of neutrophils in innate immunity: a systems biology-level approach. Wiley Interdiscip Rev Syst Biol Med 2009;1:309-333.

-6 Nathan C: Points of control in inflammation. Nature 2002;420:846-852. 
7 McLendon MK, Apicella M, Allen L-AH: Francisella tularensis: taxomony, genetics and immunopathogenesis of a potenial agent of biowarfare. Annu Rev Microbiol 2006;60: 167-185.

8 Schricker RL, Eigelsbach HT, Mitten JO, Hall WC: Pathogenesis of tularemia in monkeys aerogenically exposed to Francisella tularensis 425. Infect Immun 1972;5:734-744.

-9 Hall WC, Kovatch RM, Schricker RL: Tularemic pneumonia: pathogenesis of the aerosolinduced disease in monkeys. J Pathol 1973; 110:193-201

10 Hall JD, Woolard MD, Gunn BM, Craven RR, Taft-Benz S, Frelinger JA, Kawula TH: Infected-host-cell repertoire and cellular response in the lung following inhalation of Francisella tularensis Schu S4, LVS, or U112. Infect Immun 2008;76:5843-5852.

11 Bosio CM, Elkins KL: Susceptibility to secondary Francisella tularensis live vaccine strain infection in B-cell-deficient mice is associated with neutrophilia but not with defects in specific T-cell-mediated immunity. Infect Immun 2001;69:194-203.

-12 Malik M, Bakshi CS, McCabe K, Catlett SV, Shah A, Singh R, Jackson PL, Gaggar A, Metzger DW, Melendez JA, Blalock JE, Sellati TJ: Matrix metalloproteinase 9 activity enhances host susceptibility to pulmonary infection with type A and B strains of Francisella tularensis. J Immunol 2007;178:1013-1020.

13 Allen L-AH: Editorial: Leukocytes in tularemia - so many cells, so little time. J Leukoc Biol 2013;93:641-644.

14 Allen L-AH: Neutrophils: potential therapeutic targets in tularemia? Front Cell Infect Microbiol 2013;3:109.

15 McCaffrey RL, Allen L-AH: Pivotal advance: Francisella tularensis evades killing by human neutrophils via inhibition of the respiratory burst and phagosome escape. J Leukoc Biol 2006;80:1224-1230.

-16 McCaffrey RL, Schwartz JT, Lindemann SR, Moreland JG, Buchan BW, Jones BD, Allen L-AH: Multiple mechanisms of NADPH oxidase inhibition by type A and type B Francisella tularensis. J Leukoc Biol 2010;88:791-805.

-17 Schwartz JT, Barker JH, Kaufman J, Fayram DC, McCracken JM, Allen L-AH: Francisella tularensis inhibits the intrinsic and extrinsic pathways to delay constitutive apoptosis and prolong human neutrophil lifespan. J Immunol 2012;188:3351-3363.

18 Witko-Sarsat V, Pederzoli-Ribeil M, Hirsh E, Sozzani S, Cassatella MA: Regulating neutrophil apoptosis: new players enter the game. Trends Immunol 2011;32:117-124.

19 Geering B, Simon HU: Peculiarities of cell death mechanisms in neutrophils. Cell Death Differ 2011;18:1457-1469.

-20 Schwartz JT, Bandyopadhyay S, Kobayashi SD, McCracken J, Whitney AR, DeLeo FR, Allen L-AH: Francisella tualrensis alters hu- man neutrophil gene expression: insights into the molecular basis of delayed neutrophil apoptosis. J Innate Immun 2013;5:51245136.

21 Rossi AG, Sawatzky DA, Walker A, Ward C, Sheldrake TA, Riley NA, Caldicott A, Martinez-Losa M, Walker TR, Duffin R, Gray M, Crescenzi E, Martin MC, Brady HJ, Savill JS, Dransfield I, Haslett C: Cyclin-dependent kinase inhibitors enhance the resolution of inflammation by promoting inflammatory cell apoptosis. Nat Med 2006;12:1056-1064.

22 Leitch AE, Haslett C, Rossi AG: Cyclin-dependent kinase inhibitor drugs as potenital novel anti-inflammatory and pro-resolution agents. Br J Pharmacol 2009;158:1004-1016.

23 Bandyopadhyay S, Long ME, Allen L-AH: Differential expression of microRNAs in Francisella tularensis-infected human macrophages: miR-155-dependent downregulation of Myd 88 inhibits the inflammatory response. PLoS One 2014;9:e109525.

24 Nauseef WM: Isolation of human neutrophils from venous blood. Methods Mol Biol 2007; 412:15-20.

25 Rosser BG, Gores GJ: Cellular in vivo assay of calpain activity using a fluorescent substrate. Application to study of anoxic liver injury. Methods Mol Biol 2000;144:245-259.

26 Waterhouse NJ, Steel R, Kluck R, Trapani JA: Assaying cytochrome $c$ translocation during apoptosis. Methods Mol Biol 2004;284:307313.

27 Allen L-AH: Immunofluorescence and confocal microscopy of neutrophils. Methods Mol Biol 2007;412:273-287.

28 Maianski NA, Geissler J, Srinivasula SM, Alnemri ES, Roos D, Kuijpers TW: Functional characterization of mitochondria in neutrophils: a role restricted to apoptosis. Cell Death Differ 2004;11:143-153.

29 Maianski NA, Roos D, Kuijpers TW: Bid truncation, Bid/Bax targeting to the mitochondria, and caspase activation associated with neutrophil apoptosis are inhibited by granulocyte colony-stimulating factor. J Immunol 2004;172:7024-7030.

30 Esposti MD: Mitochondria in apoptosis: past, present and future. Biochem Soc Trans 2004; 32:493-495

- 31 O'Connor CL, Anguissola S, Huber HJ, Dussmann $\mathrm{H}$, Prehn JH, Rehm M: Intracellular signaling dynamics during apoptosis execution in the presence or absence of X-linkedinhibitor-of-apoptosis-protein. Biochim Biophys Acta 2008; 1783:1903-1913.

32 van Raam BJ, Drewniak A, Groenewold V, van den Berg TK, Kuijpers TW: Granulocyte colony-stimulating factor delays neutrophil apoptosis by inhibition of calpains upstream of caspase-3. Blood 2008;112:2046-2054.

33 Altznauer F, Conus S, Cavalli A, Folkers G, Simon H-U: Calpain-1 regulates Bax and subsequent Smac-dependent caspase-3 activa- tion in neutrophil apoptosis. J Biol Chem 2004;279:5947-5957.

- 34 Kobayashi S, Yamashita K, Takeoka T, Ohtsuki T, Suzuki Y, Takahashi R, Yamamoto K, Kaufmann SH, Uchiyama T, Sasada M, Takahashi A: Calpain-mediated X-linked inhibitor of apoptosis degradation in neutrophil apoptosis and its impairment in chronic neutrophilic leukemia. J Biol Chem 2002;277: 33968-33977.

35 Witko-Sarsat V, Mocek J, Bouayad D, Tamassia N, Ribeil J-A, Candalh C, Davezac N, Reuter N, Mouthon L, Hermine O, Pederzoli-Ribeil M, Cassatella MA: Proliferating cell nuclear antigen acts as a cytoplasmic platform controlling human neutrophil survival. J Exp Med 2010;207:2631-2645.

36 Milot E, Filep JG: Regulation of neutrophil survival/apoptosis by Mcl-1. Sci World J 2011;11:1948-1962.

37 Maianski NA, Mul FPJ, van Buul JD, Roos D, Kuijpers TW: Granulocyte colony-stimulating factor inhibits the mitochondria-dependent activation of caspase- 3 in neutrophils. Blood 2002;99:672-679.

38 Gardai SJ, Hildeman DA, Frankel SK, Whitlock BB, Frasch SC, Borregaard N, Marrack P, Bratton DL, Henson PM: Phosphorylation of Bax Ser184 by Akt regulates its activity and apoptosis in neutrophils. J Biol Chem 2004; 279:21085-21095.

-39 Simmons MJ, Fan G, Zong W-X, Degenhardt K, White E, Gelinas C: Bfl-1/A1 functions, similar to Mcl-1, as a selective tBid and Bax antagonist. Oncogene 2008;27:1421-1428.

40 Renault TT, Chipuk JE: Death upon a kiss: mitochondrial outer membrane composition and organelle communication govern sensitivity to Bak/Bax-dependent apoptosis. Chem Biol 2014;21:114-123.

41 Momeni HR: Role of calpain in apoptosis. Cell J 2011;13:65-72.

42 Srinivasa SM, Gupta S, Datta P, Zhang Z, Hegde R, Cheong N, Fernandes-Alnemri T, Alnemri ES: Inhibitor of apoptosis proteins are substrates for the mitochondrial serine protease Omi/Htra2. J Biol Chem 2003;278: 3146-31472.

43 Koedel U, Frankenberg T, Kirschnek S, Obermaier B, Häcker H, Paul R, Häcker G: Apoptosis is essential for neutrophil functional shutdown and determines tissue damage in experimental pneumococcal meningitis. PLoS Pathog 2009;5:e1000461.

44 Wang K, Hampson P, Hazeldine J, Krystof V, Strnad M, Pechan P, M J: Cyclin-dependent kinase 9 activity regulates neutrophil spontaneous apoptosis. PLoS One 2012;7:e30128.

45 Leitch AE, Lucas CD, Marwick JA, Duffin R, Haslett C, Rossi AG: Cyclin-dependent kinases 7 and 9 specifically regulate neutrophil transcription and their inhibition drives apoptosis to promote resolution of inflammation. Cell Death Differ 2012;19:1950-1961. 